\title{
Monitoring Body Fluids in Textiles: Combining Impedance and Thermal Principles in a Printed, Wearable and Washable Sensor
}

Manoj Jose ${ }^{1,2,}$ Gilles Oudebrouckx ${ }^{1,2,}$ Seppe Bormans ${ }^{1,2}$, Paula Veske ${ }^{3}$, Ronald Thoelen ${ }^{1,2}$ and Wim Deferme ${ }^{1,2, *}$

\footnotetext{
${ }^{1}$ Hasselt University, Institute for Materials Research (IMO-IMOMEC) 1, 3590 Diepenbeek, Belgium

${ }^{2}$ IMEC, Division IMOMEC, Wetenschapspark 1, B-3590 Diepenbeek, Belgium

${ }^{3}$ Centre for Microsystems Technology (CMST), IMEC and Ghent University, Technologiepark 126, 9052 Gent, Belgium

*Correspondence: wim.deferme@uhasselt.be
}

\section{Supplementary Section}

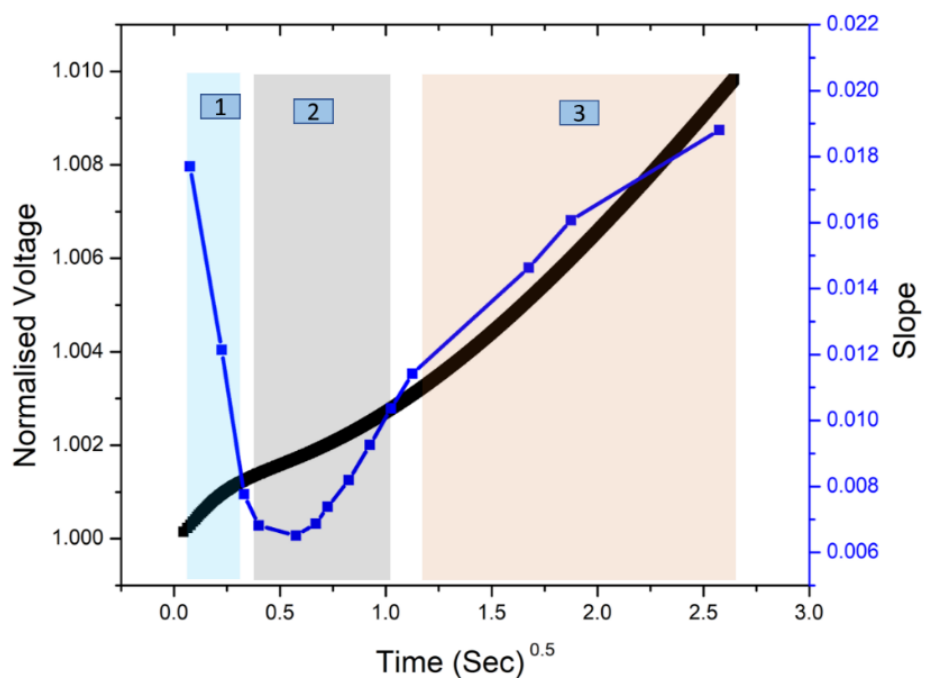

Figure S-1 Transient heating of the sensor which is fully wet and the slope varaitions corresponding to time zones.

The transient heating curve (normalised resistance vs time to the power 0.5) is plotted and the slope for a time interval of $0.05 \mathrm{~s}^{0.5}$ at different time zones is calculated. After that, the slope value is plotted against the time scale as shown in Figure S-1. Zone 1 indicates the encapsulation region of the sensor and it decreases as it reaches the liquid layer. Zone 2 indicates that the liquid is in the sensor surface. Zone 3 indicates the liquid-air interface and then air. As air has a very low thermal effusivity, the slope of the curve starts to increase. In this measurement, the water content measurement needs approximately 1 second of heating. 


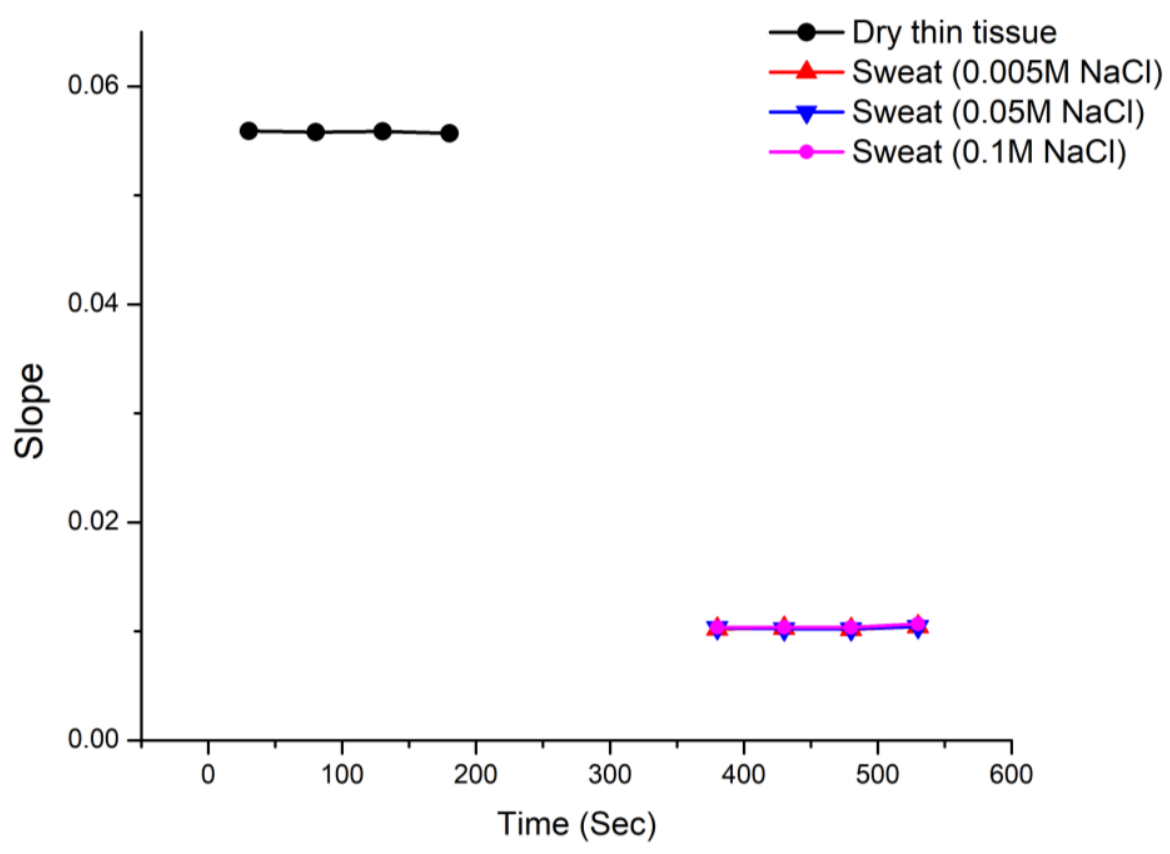

Figure S-2 TPS measurements of sweat with different molar NaCl concentrations (of equal volume) dropped on a thin tissue. This shows that there is no significant influece or impact on its readings for ionic concentrations.

This experiment is designed to show the minor deviations in the TPS sweat volume measurements (in figure 9B) are not arising from the variations in the $\mathrm{NaCl}$ concentrations. The aforementioned variations are attributed to the dropping and spreading dynamics of the liquid drop. Here the sensor is covered with thin tissue and equal volumes of sweat with different $\mathrm{NaCl}$ concentrations are dropped on it and the sensor response is recorded. The experiment results (in Figure S-2) show that the $\mathrm{NaCl}$ concentration in the range $0.005-0.1 \mathrm{M}$ has no effect on the sweat volume measurements while using the TPS measurement technique. 